\title{
Ziviler Ungehorsam: ein aktiver Beitrag zur Ent- wicklung der demokratischen Kultur in der FMH
}

Yvonne Gilli, Werner Eugster, Konrad Schiess, Jakob Schenk, Karsten Beer

Im Namen des Verwaltungsrates der xundart AG
Die Ärztenetzwerke xundart, säntiMed und Murgmed haben mit grosser Mehrheit ihrer Mitglieder beschlossen, der FMH den eingeforderten Beitrag zur Kampagnenfinanzierung der Abstimmung zur KVGRevision vom 17. Juni 2012 (Managed-Care-Vorlage) $\mathrm{zu}$ verweigern. Dazu ziehen sie einen Betrag von 30 Franken vom FMH-Jahresbeitrag ab, der durch die kantonale Ärztegesellschaft im Namen der FMH in Rechnung gestellt wird. Sie verstehen diese Handlung als zivilen Ungehorsam gegenüber einer Organisation, die offensichtlich Mühe hat, die Interessenvielfalt ihrer verschiedenen Fachgesellschaften unter ihrem Dach demokratisch und transparent zusammenzuführen. Die Meinungsbildung zu Managed Care war für uns ein Beispiel für eine Kultur, die auf intransparenter Vertretung eigennütziger Interessen, Macht und Unwissen basierte, statt auf demokratischer, offener und kontrovers gelebter Entscheidungsfindung.

Die FMH-Spitze unter dem Präsidium von Jacques de Haller begleitete die Gesetzgebung zur KVGRevision seit Beginn der Beratung in den Gesundheitskommissionen von National- und Ständerat. Jacques de Haller betonte wiederholt und öffentlich, dass die FMH Managed Care unterstütze, insbesondere die Wortfassungen zu Gatekeeping und Budgetmitverantwortung in der Gesetzesvorlage. Wir müssen es den FMH-Verantwortlichen überlassen, ob die Kehrtwende, vollzogen durch die Ärztekammer, die eine Urabstimmung einforderte, einer halbherzigen Unterstützung oder einer unsorgfältigen Prozessbegleitung der Mitglieder der Ärztekammer zuzuschreiben ist. Grundsätzlich aber anerkennen wir, dass die Urabstimmung ein gutes basisdemokratisches Element ist, um die Meinung der FMH-Basis zu erfahren.

\section{Die Formulierung der Urabstimmungsfrage}

Welche Frage wurde uns in der Urabstimmung vorgelegt?
Zum Zeitpunkt der Urabstimmung war der definitive Gesetzestext noch nicht verabschiedet. Hingegen war absehbar, in welcher Bandbreite sich der differenzierte Selbstbehalt für Patientinnen und Patienten bewegen konnte. Ebenfalls bekannt war das Verständnis der Räte zur gewählten Formulierung der Budgetmitverantwortung. Die Vertragsfreiheit war ebenso wenig Bestandteil der Vorlage wie der Datenschutz. Der aktuelle Stand der Gesetzesformulierung zum Zeitpunkt der Urabstimmung wurde den Mitgliedern vorenthalten. Die Wortwahl der für die Urabstimmung formulierten Frage suggerierte untragbare negative Konsequenzen und ist klar als manipulativ zu bezeichnen.

In der Rückschau erstaunt und alarmiert es uns, dass wir die Urabstimmung der FMH zu Managed Care überhaupt «geschluckt haben», die mit dem zu verabschiedenden Gesetz wenig Übereinstimmung zeigte. Selbstkritisch müssen wir uns heute die Frage stellen, warum wir nicht bereits zu jenem Zeitpunkt dieses manipulative Vorgehen öffentlich kritisierten. Die aktuelle Einforderung der Mitfinanzierung der professionellen Kampagne durch die FMH an die Adresse der Hausärzte und Hausärztinnen sowie der Netzwerkärzte und -ärztinnen gibt uns eine letzte Gelegenheit, unsere Stimme zu erheben. Wann, wenn nicht jetzt, signalisieren wir deutlich und öffentlich, dass wir als Mitglieder der FMH äusserst besorgt sind über die von uns als undemokratisch, intransparent und manipulativ erlebte Kultur innerhalb unserer Standesorganisation?

Vorgängig zu unserem Entschluss zu zivilem Ungehorsam befragten wir die Mitglieder unseres Netzwerks in einem Rundmail zu ihrer Haltung in dieser Frage. Wir luden auch den Dachverband der Ostschweizer Netzwerke vmco ein, die Mitglieder der darin zusammengeschlossenen Netzwerke zu befragen. Die Rückmeldungen der Mitglieder der Netzwerke xundart (SG), säntiMed (SG, AR) und Murgmed (TG) war klar: Der Schritt zum zivilen Ungehorsam wird
Korrespondenz:

Dr. med. Yvonne Gilli xundart AG Obere Bahnhofstrasse 49 CH-9500 Wil info[at]xundart.ch

\section{Stimmzettel / Bulletin de vote / Scheda di voto}

Wollen Sie den folgenden Antrag annehmen?

Verbindung der Schweizer Arztinnen und Arzte Federation des medecins suisses Swiss Medical Asrocistion

«Die FMH setzt sich konsequent gegen eine vom Parlament geplante Abschaffung der freien Arztwahl mit dem so genannt differenzierten Selbstbehalt, zwingende Budgetmitverantwortung, Vertragsfreiheit und Aushöhlung des Patientengeheimnisses ein. Die FMH setzt sich aktiv und nachweislich für dieses Ziel ein, was die Unterstützung eines allfalligen Referendums gegen eine derart gestaltete Managed-Care-Vorlage beinhaltet.

\section{JA $\square \quad$ NEIN $\square$}


mit grosser Mehrheit befürwortet. Einige Führungsmitglieder weiterer vmco-Netzwerke äusserten ihre Befürchtungen und interpretierten die Verweigerung der Bezahlung des Kampagnenbeitrages von 30 Franken als «schlechtes Verlierertum» oder als desintegrative Massnahme, die zur Spaltung der FMH führen könnte. Ihnen sei Dank für ihre Kritik. Wir nehmen sie zum Anlass, unsere Gedanken und unser Verständnis zum gefassten Beschluss darzulegen.

\section{Warum »ziviler Ungehorsam»?}

Für uns ist die Verweigerung der Bezahlung der 30 Franken, das heisst die simple Weigerung, die Gegenkampagne mitzufinanzieren, nicht gleichzusetzen mit einer drohenden Abspaltung von der FMH im Gegenteil. Diese Verweigerung ist für uns ein konkretes und symbolisches Zeichen für die Initiierung eines zukunftsbezogenen und echt demokrati-

\section{Stellungnahme der FMH}

\begin{abstract}
Mitgliederbeiträge à la carte gibt es nicht
Die Mehrheit der Mitglieder der Ärztenetzwerke xundart, säntiMed und Murgmed hat beschlossen, den Beitrag zur Finanzierung der Managed-Care-Abstimmungskampagne vom FMH-Mitgliederbeitrag abzuziehen. Doch Mitgliederbeiträge à la carte sind in der FMH nicht möglich. Es ist im Verein wie beim Staat: Wenn sich die Mehrheit des Volkes in der Abstimmung für den Kauf des FA-18 ausspricht, zahlen in der Folge alle Bürgerinnen und Bürger mit ihren Steuern für die Beschaffung des Jets.

Zum Hintergrund: Die Mehrheit der Ärztekammer hatte im Mai 2011 die Auffassung vertreten, es sei zu früh für die FMH, definitiv Position zur Managed-Care-Vorlage zu beziehen. Gegen diesen Beschluss wurde die Urabstimmung verlangt. Wie bei eidgenössischen Referenden gilt auch für Urabstimmungen innerhalb der $\mathrm{FMH}$, dass beide Seiten, Befürworter und Gegner, ihre Standpunkte fair und ausgewogen präsentieren können, wie dies im Vorfeld der Urabstimmung auch geschehen ist. Mit einer Zweidrittelmehrheit gegen die Vorlage hat die Urabstimmung ein klares Resultat ergeben. Im Anschluss haben sich alle Gremien, vom FMH-Zentralvorstand über die Delegiertenversammlung bis hin zur Ärztekammer demokratisch an diesem Mehrheitsentscheid der Mitglieder orientiert. Folgerichtig hat die Ärztekammer im Oktober 2011 nach ausführlicher Diskussion ein Kampagnenbudget beschlossen und auch den Antrag der SGAM mit 128 gegen 26 Stimmen bei 4 Enthaltungen klar angenommen, dass alle Mitgliederkategorien von 1 bis 4, die VSAO-Mitglieder eingeschlossen, den gleichen Sonderbeitrag von 30 Franken bezahlen sollen (vgl. Ärztekammerprotokoll, SÄZ 51-52/2011 [1]). Alle FMH-Mitglieder, die mit diesem Entscheid nicht einverstanden waren, hätten mit einer Urabstimmung das Referendum gegen den Mitgliederbeitrag für 2012 ergreifen können. Auf diese demokratische Referendumsmöglichkeit zu verzichten und im Nachhinein den Mitgliederbeitrag teilweise zu verweigern, ist im Vereinsrecht nicht möglich. Mitgliederbeiträge à la carte gibt es nicht.
\end{abstract}

\section{Hanspeter Kuhn, Fürsprecher, Leiter FMH-Rechtsdienst}

1 Bütikofer AG. Protokoll der ausserordentlichen Ärztekammer. Schweiz Ärztezeitung. 2011;92(51/52): 1987-98. Zitat S. 1991: «Der Antrag der SGAM (Nr. 5, Finanzierung des Referendums mit einem Sonderbeitrag, der für die Kategorien 1 bis 430 Franken beträgt) wird von den Delegierten mit 128 zu 26 Stimmen und 4 Enthaltungen klar angenommen. Der Antrag des ZV/Referendumsorganisationen (Nr. 3, modifiziert entsprechend Antrag SGAM) wird mit 130 zu 4 Stimmen und 18 Enthaltungen klar angenommen.» schen Dialogs. Wir haben bewusst den Begriff des «zivilen Ungehorsams» gewählt. Die vertiefte Auseinandersetzung mit diesem Begriff lohnt sich, gerade im Zusammenhang mit demokratischer Kultur. Immerhin haben sich von Mahatma Gandhi bis Martin Luther King grosse Männer des zivilen Ungehorsams bedient, nicht aus Eigeninteresse und nicht aus persönlicher Kränkung, sondern aus tiefer Besorgnis um die Gesellschaft und mit dem Bewusstsein, dass ziviler Ungehorsam ein unabdingbares Instrument einer demokratischen Kultur darstellt, das zur Entwicklung gerechter Strukturen notwendig ist.

Als Vater des zivilen Ungehorsams gilt der Amerikaner Henry David Thoreau. Er lebte im 19. Jahrhundert und leistete einen essentiellen Beitrag zu wichtigen demokratischen Reformschritten, sowohl durch seine konkrete Lebensführung als auch durch sein Werk. Die Rechtfertigung zivilen Ungehorsams ist Gewissenssache. Dazu Thoreau: «Muss der Bürger auch nur einen Augenblick, auch nur ein wenig sein Gewissen dem Gesetzgeber überlassen? Wozu hat dann jeder Mensch ein Gewissen? Ich finde, wir sollten erst Menschen sein, und danach Untertanen» [1]. Mahatma Gandhi wird zu diesem Thema zitiert mit den Worten: «In Gewissensangelegenheiten darf es kein Mehrheitsrecht geben» [2]. Viele Theoretiker thematisierten in ihren Werken den zivilem Ungehorsam, so zum Beispiel auch John Rawls in seiner wegweisenden Gerechtigkeitstheorie [3]. Ihnen gemeinsam ist, dass ziviler Ungehorsam immer auch gegen inneren Widerstand geschieht, da er die moralische Pflicht zu Gesetzesgehorsam bricht. Weder Eigennutz noch das Bedürfnis nach Unruhestiftung legitimieren zivilen Ungehorsam. Es ist die innere Überzeugung, eben das Gewissen, das sich gedankenlosem Mitläufertum widersetzt. Diese grundsätzliche Treue zum System verbunden mit einer Wachheit gegenüber der eigenen Überzeugung ist Voraussetzung für die Weiterentwicklung und Lebendigkeit jeder demokratischen Kultur, sei sie in der grossen Politik, sei sie in der vergleichsweise kleinen Standeskultur, wie sie in der FMH gelebt wird.

Unsere Verweigerung zur Bezahlung dieses Beitrages soll ein Zeichen setzen, dass es uns mit einer neuen und demokratischeren Kultur ernst ist und wir uns auch tatkräftig für dieses Ziel einsetzen werden. In diesem Sinn freuen wir uns auf die konstruktive und zukunftsgerichtete Zusammenarbeit mit dem neugewählten Präsidium und Zentralvorstand unserer Standesorganisation.

\section{Literatur}

1 Thoreau HD. Über die Pflicht zum Ungehorsam gegen den Staat. Zürich: Diogenes; 2012.

2 www.mkgandhi.org

3 Rawls J. Eine Theorie der Gerechtigkeit, Frankfurt a. M.: Suhrkamp; 1975. 